\section{Identification of microorganisms grown in blood culture flasks using liquid chromatography-tandem mass spectrometry}

\author{
Erwin M Berendsen', Evgeni Levin², René Braakman', Debora van der Riet-van \\ Oeveren', Norbert JA Sedee' \& Armand Paauw ${ }^{*}, 1$
}

\begin{abstract}
Aim: Bloodstream infections are a common cause of disease and a fast and accurate identification of the causative agent or agents of bloodstream infections would aid the start of adequate treatment. Materials \& methods: A liquid chromatography-tandem mass spectrometry (LC-MS/MS) shotgun proteomics method was developed for the identification of bacterial species directly from blood cultures that were simulated by inoculating blood culture bottles with single or multiple clinically relevant microorganisms. Results: Using LC-MS/MS, the single species were correctly identified in $100 \%$ of the blood cultures, whereas for polymicrobial infections, $78 \%$ of both species were correctly identified in blood cultures. Conclusion: The LC-MS/MS method allows for the identification of the causative agent of positive blood cultures.
\end{abstract}

First draft submitted: 16 March 2017; Accepted for publication: 5 June 2017; Published online: 15 September 2017

Bloodstream infections (BSIs) are associated with high morbidity and mortality [1-3]. This particularly holds true for those BSIs associated with antibiotic resistance [4]. Blood cultures are the golden standard for the detection of BSIs in patients with suspected sepsis. However, when a blood culture instrument reports a positive blood culture, there is still a delay of $48 \mathrm{~h}$ in the species identification and susceptibility testing, despite the number of colony-forming units (CFU) is ranging from $10^{7}$ to $10^{9} \mathrm{CFU} / \mathrm{ml}[5]$. Due to this delayed availability of data to the clinician, such methods have only a limited value on the clinical management [6]. Another complicating factor is that BSIs can be caused by more than one microorganism (polymicrobial) with estimates ranging from 6 to 34\%, of the BSIs being polymicrobial $[7,8]$.

Clearly, there is a need for rapid and accurate identification of the causative agent of BSIs, and moreover to be able to accurately identify the causative agents of polymicrobial infections. A faster identification of microorganisms causing BSIs would allow appropriate species-specific therapy to be started sooner, which could improve patient outcome and reduce the risk of potential development of resistance $[9,10]$. The latter would stimulate prudent use of antimicrobial agents, which can help to reduce the selective pressure that enables the development and spread of antimicrobial resistance.

'Department of CBRN Protection, Netherlands Organization for Applied Scientific Research TNO, Lange Kleiweg 137, 2288 GJ Rijswijk, The Netherlands

2Department of Microbiology \& Systems Biology, Netherlands Organization for Applied Scientific Research TNO, Utrechtseweg 48, 3704HE Zeist, The Netherlands

*Author for correspondence: armand.paauw@tno.nl

\section{KEYWORDS}

- bloodstream infection

- identification • LC-MS/MS 
Multiple methods have been developed that shorten the time to identify the causative agent of BSIs; however, these methods are often targeted approaches (PCR or immunoassays) or lack specificity (matrix-assisted laser desorption ionization - time-of-flight mass spectrometry [MALDI-TOF MS]) for direct identification from positive blood cultures. MALDI-TOF MS is used for rapid identification of the causative agent of positive blood cultures and this method can also be applied directly to the positive blood culture, without the need of a cultivation step [9]. However, MALDI-TOF MS is not always able to discriminate between closely related species $[11,12]$. Furthermore, because the identification by MALDI-TOF MS depends on the comparison of MS profiles to a reference library, identification of multiple species in one sample is cumbersome [13,14].

An approach that is based on protein sequence identification would provide additional and more accurate identification. A liquid chromatography-tandem mass spectrometry (LC-MS/MS)-based shotgun proteomics approach allows protein sequence-based identification that potentially identifies any protein that is expressed by a microorganism. The use of an LC-MS/MS method was previously applied for the identification of various cultured microorganisms [15]. Using this LC-MS/MS method, proteins of pure cultures of a microorganism are digested with trypsin, generating peptides of which the amino acid sequence is determined based on their measured masses, the masses of fragmentation product ions and their comparison with in silico trypsin digests and corresponding predicted fragments of a protein database. From these peptide sequences, not only proteins of interest can be identified, also the microorganism itself can be specified to, at least, species level $[16,17]$. Recent developments in proteomic analysis using LC-MS/MS reduced the time to result, making it possible to analyze a sample within $6 \mathrm{~h}$ [18].

The aim of this study was to determine whether shotgun proteomics could improve identification of causative agents of BSIs, both single species and polymicrobial, directly from blood culture bottles. The presence of proteins derived from the injected blood and the growth media could interfere with the identification of the microorganism(s) from a blood culture. Therefore, we developed a sample preparation method that preferentially decreases the number of nonmicrobial proteins in the sample. As a result, our method allows an accurate identification of microorganisms growing in positive blood cultures.

\section{Materials \& methods \\ - BSIs: tested isolates}

The microorganisms used for cultivations in blood culture flasks, to simulate BSIs, are listed in Table 1. A total of 34 bacterial isolates belonging to 27 bacterial species, and 1 yeast strain, were used for spiking in order to obtain blood cultures, as described below. This collection of microorganisms represents a clinically relevant selection of commonly found BSIs and two simulants for Burkholderia pseudomallei, a common cause of community-acquired BSI in Southeast Asia [19]. Additionally, strains were included for which the identification by MALDI-TOF MS is known to be challenging, namely Escherichia coli, Shigella sp., streptococci and coagulasenegative staphylococci [20]. The bacteria and yeast were cultivated (described below) to mimic monomicrobial infections, and for a selection of bacteria and yeast also as polymicrobial infections. The simulated polymicrobial infections are listed in Table 2.

\section{- Culture conditions}

From the $-80^{\circ} \mathrm{C}$ stock, the bacteria and yeast were recovered on blood agar plates and cultured for at least $24 \mathrm{~h}$ at $35^{\circ} \mathrm{C}$ and, depending on the species, in the presence of $5 \% \mathrm{CO}_{2}$. Before inoculation of the blood culture flasks, the isolates were regrown on a blood agar plate for $24 \mathrm{~h}$ at $35^{\circ} \mathrm{C}$ in the presence of $5 \% \mathrm{CO}_{2}$ when required. For all strains, suspensions of $1 \times 10^{3}-1 \times 10^{4} \mathrm{CFU} / \mathrm{ml}$ were made in Dulbecco's phosphate buffered saline (DPBS; Lonza, Verviers, Belgium). Subsequently, $1 \mathrm{ml}$ of suspension per species, for the mono- and polymicrobial infections, was injected in an aerobic and anaerobic BACTEC blood culture flask, respectively (Beckton Dickinson, Breda, The Netherlands). These blood culture flasks were injected with $9 \mathrm{ml}$ of $50 \%$ sheep erythrocytes (DivBioScience, Ulvenhout, The Netherlands) to mimic blood culture conditions. For each bacterium and yeast, and for the polymicrobial infections, and two negative controls, one aerobic and one anaerobic flask was inoculated. The inoculated flasks for mono- and poly-microbial infections were incubated for $16 \mathrm{~h}$ at $35^{\circ} \mathrm{C}$. Subsequently, $1 \mathrm{ml}$ of 
Table 1. Strains used in this study.

\begin{tabular}{|c|c|c|c|}
\hline Strain no. & Aliases & Genus & Species \\
\hline BM1501 & & Acinetobacter & baumannii \\
\hline BM1504 & & Acinetobacter & baumannii \\
\hline BM658 & DSMZ 30007 & Acinetobacter & baumannii \\
\hline BM642 & DSMZ 21774 & Burkholderia & oklahomensis \\
\hline BM902 & DSMZ 13276 & Burkholderia & thailandensis \\
\hline BM116 & & Citrobacter & freundii \\
\hline BM268 & & Enterococcus & faecalis \\
\hline BM656 & ATCC 14506 & Enterococcus & faecalis \\
\hline BM1368 & E1071 & Enterococcus & faecium \\
\hline BM1518 & & Enterococcus & faecium \\
\hline BM1381 & ECOR-04 & Escherichia & coli \\
\hline BM1399 & ECOR-22 & Escherichia & coli \\
\hline BM768 & $\mathrm{K} 12 \mathrm{DH} 5 \alpha$ & Escherichia & coli \\
\hline BM916 & DSMZ 13698 & Escherichia & fergusonii \\
\hline BM636 & ATCC 43816 & Klebsiella & pneumoniae \\
\hline BM365 & & Listeria & monocytogenes \\
\hline BM766 & DSMZ 22644 & Pseudomonas & aeruginosa \\
\hline BM256 & & Salmonella & enteritidis \\
\hline BM255 & & Salmonella & typhimurium \\
\hline BM917 & DSMZ 7532 & Shigella & boydii \\
\hline BM1142 & & Shigella & dysenteriae \\
\hline BM918 & DSMZ 4782 & Shigella & flexneri \\
\hline BM919 & DSMZ 5570 & Shigella & sonnei \\
\hline BM494 & ATCC 43300 & Staphylococcus & aureus \\
\hline BM495 & ATCC 25923 & Staphylococcus & aureus \\
\hline BM493 & ATCC 12228 & Staphylococcus & epidermidis \\
\hline BM934 & DSMZ 20322 & Staphylococcus & simulans \\
\hline BM265 & & Streptococcus & agalactiae \\
\hline BM266 & & Streptococcus & dysgalactiae \\
\hline BM264 & & Streptococcus & pyogenes \\
\hline BM932 & DSMZ 20352 & Staphylococcus & lentus \\
\hline BM933 & DSMZ 20260 & Staphylococcus & cohnii \\
\hline BM935 & DSMZ 20326 & Staphylococcus & capitis \\
\hline BM357 & & Streptococcus & mutans \\
\hline BM659 & ATCC 14053 & Candida & albicans \\
\hline
\end{tabular}

positive blood culture was subtracted from the medium and used for sample preparation for LC-MS/MS analysis. Exceptions were made for slow growing microorganisms: Candida albicans, Staphylococcus lentus, Staphylococcus cohnii, Staphylococcus capitis and Streptococcus mutans. For these bacteria the blood culture flasks were incubated for $48 \mathrm{~h}$ and at time points $24 \mathrm{~h}$ and $48 \mathrm{~h}, 1 \mathrm{ml}$ of the blood culture was subtracted from the medium for sample preparation for LC-MS/MS analysis. The species Acinetobacter baumannii, Burkholderia oklahomensis and Burkholderia thailandensis are strict aerobic bacteria and for these bacteria only aerobic flasks were inoculated.
- Sample preparation for LC-MS/MS analysis From each positive blood culture flask, $1 \mathrm{ml}$ was withdrawn from the suspension and transferred into a tube. The samples were centrifuged for $5 \mathrm{~min}$ at $21,000 \times \mathrm{g}$. Erythrocytes and proteins in the culture medium potentially interfere with the LC-MS/MS analyses. To remove such proteins as much as possible, the following steps were executed twice. Upon removal of the supernatant, $1 \mathrm{ml} \mathrm{2.5 \%}$ saponin was added, followed by mixing and 5 -min incubation at room temperature. Subsequently, the suspension was centrifuged for $5 \mathrm{~min}$ at $21,000 \times g$ followed by washing with DPBS and demineralized water for 1 min, respectively. 
RESEARCH ARTICLE Berendsen, Levin, Braakman, van der Riet-van Oeveren, Sedee \& Paauw

\begin{tabular}{|c|c|c|c|c|c|c|c|}
\hline \multirow[t]{2}{*}{ Sample } & \multirow[t]{2}{*}{ Injected microorganisms } & \multicolumn{2}{|c|}{$\begin{array}{c}\text { Number of discriminative } \\
\text { peptides detected }\end{array}$} & \multicolumn{2}{|c|}{ Correct identification } & \multicolumn{2}{|c|}{ First false-positive hit } \\
\hline & & Aerobic & Anaerobic & Aerobic & Anaerobic & Aerobic & Anaerobic \\
\hline \multirow[t]{2}{*}{1} & Enterococcus faecium & 163 & 219 & Yes & Yes & 1 & 1 \\
\hline & Staphylococcus epidermidis & 33 & 9 & Yes & No & & \\
\hline \multirow[t]{2}{*}{2} & Pseudomonas aeruginosa & 469 & 135 & Yes & Yes & 2 & 1 \\
\hline & Staphylococcus epidermidis & 5 & 98 & No & Yes & & \\
\hline \multirow[t]{3}{*}{3} & Pseudomonas aeruginosa & 165 & 5 & Yes & No & 3 & 2 \\
\hline & Streptococcus agalactiae & $341^{\ddagger}$ & $491^{\ddagger}$ & Yes & Yes & & \\
\hline & & $612^{\S}$ & $820^{\S}$ & Yes & Yes & 3 & 4 \\
\hline \multirow[t]{2}{*}{4} & Citrobacter freundii & 56 & 42 & Yes & Yes & 1 & 1 \\
\hline & Staphylococcus aureus & 53 & 34 & Yes & Yes & & \\
\hline \multirow[t]{2}{*}{5} & Staphylococcus epidermidis & 0 & 4 & No & No & 5 & 2 \\
\hline & Staphylococcus simulans & 459 & 267 & Yes & Yes & & \\
\hline \multirow[t]{2}{*}{6} & Enterococcus faecium & 142 & 191 & Yes & Yes & 2 & 1 \\
\hline & Staphylococcus simulans & 93 & 58 & Yes & Yes & & \\
\hline \multirow[t]{3}{*}{7} & Candida albicans & 0 & 0 & No & No & 2 & 1 \\
\hline & Escherichia coli & $31^{n}$ & $33^{n}$ & Yes & Yes & & \\
\hline & & $5^{\#}$ & $10^{\#}$ & Yes & Yes & 0 & 0 \\
\hline \multirow[t]{3}{*}{8} & Staphylococcus aureus & 85 & 91 & Yes & Yes & 6 & 2 \\
\hline & Streptococcus dysgalactiae & $52^{\ddagger}$ & $39^{\ddagger}$ & Yes & Yes & & \\
\hline & & $419^{t+}$ & $29^{+t}$ & Yes & Yes & 8 & 7 \\
\hline \multirow[t]{2}{*}{9} & Klebsiella pneumoniae & 32 & 33 & Yes & Yes & 1 & 1 \\
\hline & Enterococcus faecalis & 63 & 103 & Yes & Yes & & \\
\hline \multirow[t]{3}{*}{10} & Candida albicans & 0 & 0 & No & No & 1 & 1 \\
\hline & Staphylococcus epidermidis & 0 & 0 & No & No & & \\
\hline & Citrobacter freundii & 76 & 55 & Yes & Yes & & \\
\hline
\end{tabular}

After washing, the pellet was resuspended in $400 \mu \mathrm{l}$ of $100 \mathrm{mM}$ ammonium bicarbonate and incubated at $95^{\circ} \mathrm{C}$ for $10 \mathrm{~min}$. Using $0.1 \mathrm{~mm}$ silica glass beads (Sigma-Aldrich, Zwijndrecht, The Netherlands) and the Omni Bead Ruptor (LA Biosystems, Waalwijk, The Netherlands), microorganisms were ruptured by beating at $3.25 \mathrm{~m} / \mathrm{s}$ for $5 \mathrm{~min}$. Dithiothreitol (DTT; Sigma-Aldrich) and trypsin (Sigma-Aldrich) were added to final concentrations of $5 \mathrm{mM}$ and $10 \mu \mathrm{g} / \mathrm{ml}$, respectively, and samples were incubated for $1 \mathrm{~h}$ at $37^{\circ} \mathrm{C}$. Trypsin digestion was stopped by addition of formic acid to a final concentration of $0.1 \%$. Large particles were removed by centrifugation $(1 \mathrm{~min}, 10,000 \times g)$ and supernatants were filtered through a Microcon centrifugal filter device with a cut-off of $30 \mathrm{kDa}$ (Merck Millipore, Amsterdam, The Netherlands).

\section{- LC-MS/MS analysis of whole-cell lysates}

The protein digests were analyzed with LC-MS/MS using a UHPLC system (Dionex Ultimate 3000 RS pump; Thermo Fisher Scientific, Germering, Germany) coupled to a Q-TOF mass spectrometer (maXis impact, Bruker Daltonics GmbH, Bremen, Germany). The samples were injected onto an Acquity HSS T3 UHPLC column ( $1 \mathrm{~mm}$ ID $\times 150 \mathrm{~mm}$, $1.8 \mu \mathrm{m}, 100 \AA$, Waters, Etten-Leur, The Netherlands) and separated at a constant flow of $0.1 \mathrm{ml} / \mathrm{min}$, with the following binary gradient: from $100 \% \mathrm{~A}(0.2 \%$ formic acid in milli-Q $\mathrm{H}_{2} \mathrm{O}$, Sigma-Aldrich) to $50 \% \mathrm{~B}(0.2 \%$ formic acid in acetonitrile; Sigma-Aldrich) in $45 \mathrm{~min}$, followed by an increase to $80 \% \mathrm{~B}$ for $5 \mathrm{~min}$. The UHPLC system was coupled to the mass spectrometer using an electrospray 
ionization source (ESI-source, Bruker Daltonics, $\mathrm{GmbH})$. The spray voltage was set at $4.5 \mathrm{kV}$ and the temperature of the heated capillary was set to $180^{\circ} \mathrm{C}$. Eluting peptides were analyzed using the data-dependent MS/MS mode. The ten most abundant ions (charge state $2+, 3+$ and $4+$ ) in an MS spectrum $(300-1300 \mathrm{~m} / \mathrm{z}$ ) were selected for data-dependent MS/MS analysis by collisioninduced dissociation using nitrogen as the collision gas. MS/MS scans were acquired over a mass range of $100-2000 \mathrm{~m} / \mathrm{z}$.

\section{- Protein database \& peptide assignment}

To assign MS data, a custom database was constructed in a FASTA format using annotated protein sequences derived from sequenced reference genomes on the NCBI FTP server extracted on 29 August 2016, supplemented with annotated genomes of which no reference genome was available at that time. The database contained 447 entries, representing 434 bacterial species, 2 Cryptococcus sp., 6 Candida sp., and single entries of Ricin communis, Ovis aries, Bos taurus and Homo sapiens. Ovis aries was included for the identification of peptides derived from erythrocytes, Bos taurus for blood culture media and trypsin derived peptides, Homo sapiens for possible contamination and cross-reactivity and Ricin communis because the database is used to screen for potential biothreat agents, as well. Furthermore, E. coli and Shigella species were combined into a single database entry because these species are closely related in their protein content and Streptococcus agalactiae and Streptococcus dysgalactiae, as well.

From each sample the obtained MS spectra were assigned to peptides using PEAKS 7.5 and the custom database (Bioinformatics Solutions Inc., Waterloo, Canada) [21]. Furthermore, filters were used to exclude duplicate peptides and advanced missed cleavage handling. Only peptides assigned with a high degree of certainty (false discovery rate [FDR] of $<0.01 \%$ ) were used to analyze which microorganisms were present in the original sample. Next, using a Python script it was determined in which entries the measured peptide sequences occurred. Finally, for each entry the number of discriminative designated peptides were counted.

To identify the E. coli or Shigella sp. to the species level upon detection a second analysis was performed with a database consisting only of E. coli, Shigella boydii, Shigella dysenteriae, Shigella flexneri and Shigella sonnei.
Upon detection of a $S$. agalactiae or a $S$. dysgalactiae a second analysis was performed with a database consisting only of $S$. agalactiae and S. dysgalactiae.

\section{Results}

- Identification of monomicrobial infections

For a total of 33 blood cultures, mimicking monomicrobial episodes, direct identification was attempted using LC-MS/MS. The microorganisms were correctly identified in 33 of the $33(100 \%)$ aerobic blood cultures, and in 27 of the $28(96 \%)$ anaerobic blood cultures. The lowest number of identified discriminative peptides per entry was 26 for the combined entry E. coli/Shigella sp., whereas the highest number of discriminative peptides was 853 for the entry Enterococcus faecalis. Despite the presence of matrix-derived peptides, there are sufficient microorganism discriminative peptides that allow for identification (Supplementary Table 1). To determine repeatability, E. coli strain BM768 was used in four independent experiments all mimicking a monomicrobial episode. The identification was always correct with assigned peptides ranging from 51 to 100 to the combined entry E. coli/Shigella sp. and the number of $E$. coli discriminative peptides ranging from 9 to 14 in the second-stage analysis (Table 3 ). For S. cohnii and S. capitis correct identifications were obtained after $24 \mathrm{~h}$ in case of aerobic blood culture, and after $48 \mathrm{~h}$ in case of anaerobic blood culture. C. albicans was correctly identified in a $1 \mathrm{ml}$ sample taken from the aerobic flask after 48 h of incubation. In contrast, after $24 \mathrm{~h}$ of incubation identification of multiple C. albicans discriminative peptides was not successful. Furthermore, for $C$. albicans no growth was detected upon anaerobic conditions in blood culture, therefore identification was not possible (Table 3).

Of the tested microorganisms, S. lentus contained the highest number of falsely classified peptides $\left(n_{\text {peptides }}=14\right)$. Based on the results, the cut-off to identify a species (entry) was empirically established as more than 14 discriminative peptides. In most cases, the observed false classifications were peptides assigned to another species from the same genus (data not shown). False classification by assigning peptides to another species is possible because of the limited size of the custom database where not all possible mobile genetic elements are represented in each species entry. 
RESEARCH ARTICLE Berendsen, Levin, Braakman, van der Riet-van Oeveren, Sedee \& Paauw

Table 3. Identified peptides from blood culture flasks injected with microorganisms using liquid chromatography-tandem mass spectrometry.

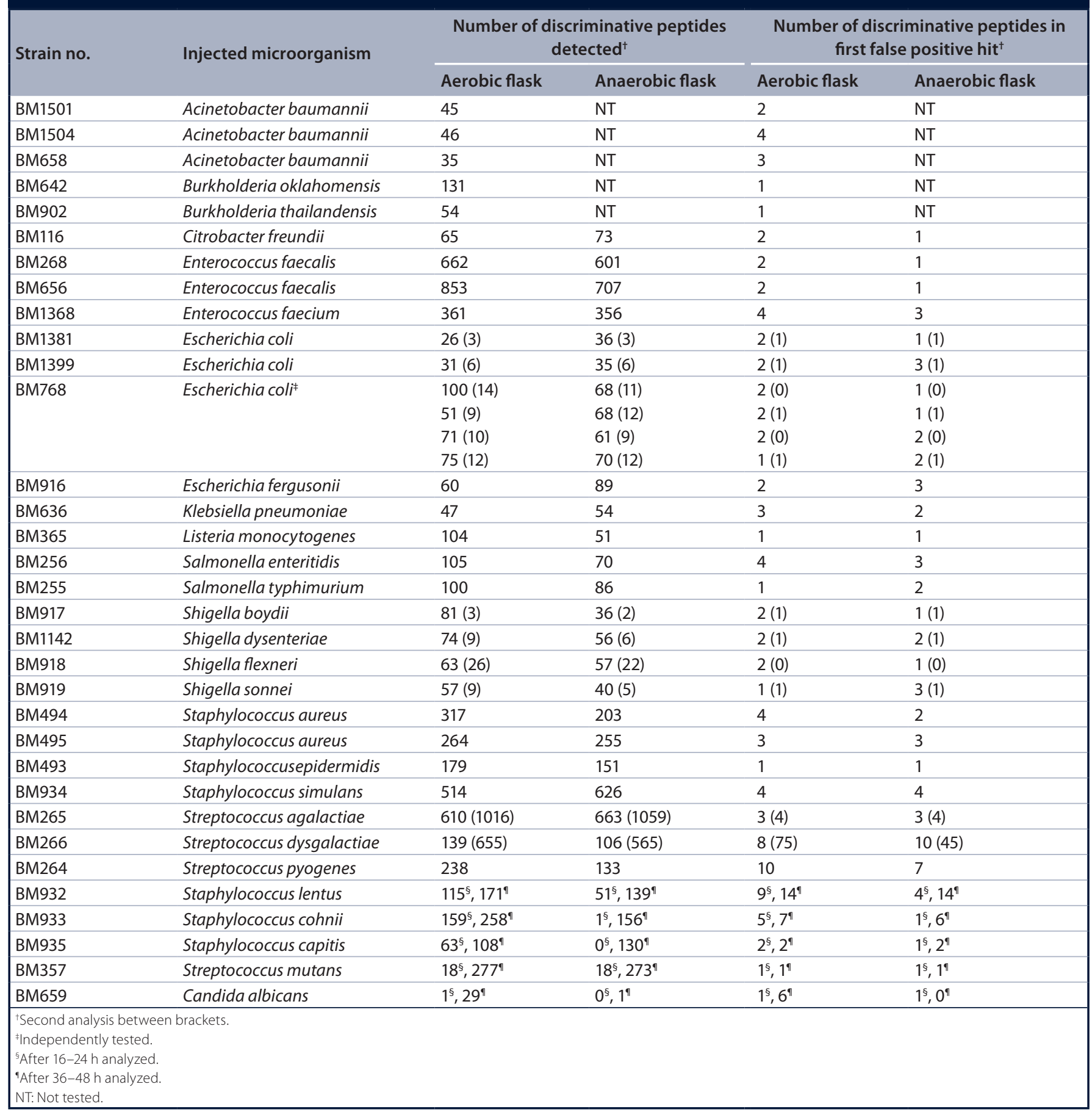

\section{- Distinction between closely related species}

The E. coli and Shigella sp. strains are genetically very closely related; therefore, there is a limited number of discriminative peptides that directly discriminates between E. coli, S. boydii, S. dysenteriae, S. flexneri and S. sonnei. Furthermore, discrimination between $S$. agalactiae and $S$. dysgalactiae was hampered due to the relative high number of peptides falsely identified as specific for these two entries, due to a database limitation. Therefore, a two-step approach was used for the identification of these strains to the species level. After initial identification 
of a strain belonging to the E. coli/Shigella entry or the $S$. agalactiae/S. dysgalactiae entry, a second search was performed against a smaller database only containing strains of the closely related species. Using this two-step approach, all tested E. coli and Shigella sp. strains were correctly discriminated from each other based on the highest number of discriminative peptides (Tables 2 \& 3). Likewise, the tested $S$. agalactiae and $S$. dysgalactiae were correctly identified (Tables 2 \& 3).

\section{- Identification of polymicrobial infections}

For polymicrobial infections it can be difficult to identify all microorganisms present in a positive blood culture. For the polymicrobial blood cultures containing two species, 7 out of 9 (78\%) were correctly identified, upon combining results of the aerobic and anaerobic blood culture (Table 3). In the remaining two spiked blood cultures with two species only one of the two species was identified. From the polymicrobial blood culture spiked with three species, only one species was identified.

\section{Discussion}

BSI is an important cause of illness and deaths. A BSI is commonly traced by a positive blood culture. Then a time-consuming process is needed that involves culture-based identification of the causative agent [22]. Speeding up identification of the causative agent(s) in a blood culture is crucial for clinical decision making and optimal antibiotic therapy. In this study, an LC-MS/MS method was developed for direct identification of microorganisms from positive blood cultures (Figure 1). This method is generic and is based on the determination of multiple discriminative peptide sequences for the identification of microorganisms causing BSI.

The LC-MS/MS method allowed for correct identification of mimicked monomicrobial blood cultures from aerobic and anaerobic blood culture flasks in 100\% (33/33) and 96\% (27/28), respectively. The result of one strain of E.coli, that was cultured four-times, and identified correctly in all cases, indicates the robustness of the method. The reported identification rates with MALDI-TOF MS directly from positive blood cultures ranged from 66 to $85.2 \%$ for monomicrobial blood cultures, which is lower compared with the $100 \%$ identification found using the LC-MS/MS method [9,23-26].

The developed LC-MS/MS method correctly identified both microorganisms in 78\% (7/9) of the nine blood cultures spiked with two microorganisms, and one of the two microorganisms in the two other spiked samples $(22 \%)$. In one case where a polymicrobial blood culture with three agents was mimicked, only one of the three microorganisms was identified using LC-MS/MS. The identification of microorganisms in polymicrobial blood samples with LC-MS/MS was potentially
Blood culture

$$
\begin{aligned}
& \text { Sample prep } \\
& \text { - Clean up } \\
& \text { - Trypsin digestion }
\end{aligned}
$$

\section{LC-MS/MS analysis}

Data analysis

Peptides

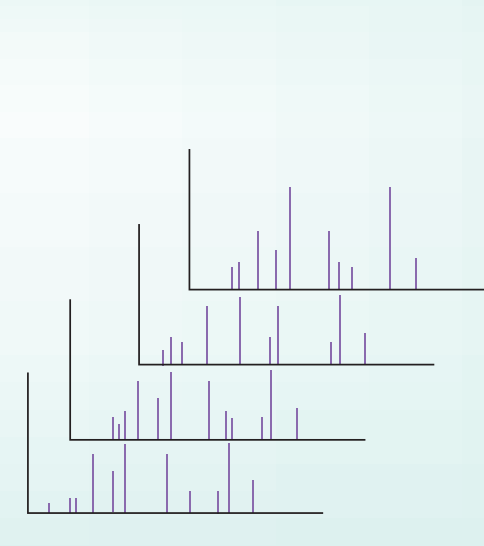

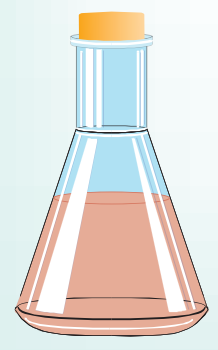

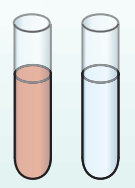

Peptides

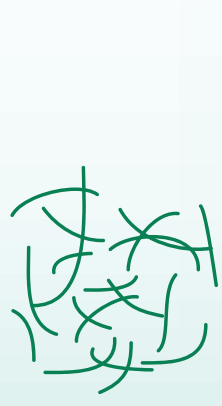

Figure 1. Schematic overview of the liquid chromatography-tandem mass spectrometry-based method for the identification of microorganisms from blood cultures.

LC-MS/MS: Liquid chromatography-tandem mass spectrometry. 
hampered by the competition between different microorganisms during growth in the blood culture flasks, where one species overgrows the other species, however this was not examined.

For polymicrobial species identification using MALDI-TOF MS directly on positive blood cultures, the reported method was able to identify both microorganisms in one out of ten polymicrobial cultures, and in five out of ten blood cultures only one of the two microorganisms was identified [9]. Similarly, in another study, in $64.5 \%$ only one of the two microorganisms was identified to the species level, whereas no correct identification was found for the other polymicrobial blood cultures [24]. The identification rate of MALDI-TOF MS for polymicrobial blood cultures is considerably lower compared with the $78 \%$ identification rate of all microorganisms, in the case of two microorganisms, using LC-MS/MS.

To identify genetically closely related species - E. coli and Shigella sp. and S. agalactiae and $S$. dysgalactiae - a two-step data analysis was developed. In the first step the closely related species were combined in a single entry, allowing for the identification of discriminative peptides for the combined species, and thereby providing a preliminary identification. In the second step, discriminative peptides were identified allowing for identification at a species level, albeit with a relatively low numbers of discriminating peptides. The relative low number of discriminative peptides for some species is potentially caused by the limited number of strains per species included in the database and by the overlap in proteomes between some genetically closely related bacterial species. Moreover, between some species genetic exchange takes place regularly, which possibly limits the number of discriminative determinants between closely related species populations. A good example is the species E. coli and the different Shigella species, which do not constitute monophyletic clades within the E. coli-Shigella phylogroup and therefore share a large part of their proteome, which is likely to limit the number of discriminative peptides per species [27-30]. The species $S$. agalactiae belongs to the haemolytic group B, while $S$. dysgalactiae is a HSC-group-C-based on Lancefield typing scheme for haemolytic Streptococci [31]. However, genetically, S. dysgalactiae is a sub-branch of $S$. agalactiae [31], which explains the large overlap in genome and protein sequences between the two species. Potentially, horizontal transfer of genes can lead to specific gene presence in strains of the same or closely related species, which could explain that some proteins are found in only a portion of the strains from one particular species. Furthermore, a possible limitation for correct identification of microorganisms is the dependency on the database. A good-quality identification depends on the size of the database, and requires good quality data in this database. Therefore, the threshold for the identification of bacterial species was empirically determined in this study.

In general, rapid unambiguous identification of microorganisms and distinction from closely related species is an analytical challenge in clinical microbiology. Often additional techniques are needed to confirm a preliminary identification. A main advantage of the LC-MS/MS method is the sequence-based identification, where identification of the species is supported by multiple discriminative peptide sequences. This allows for the unambiguous identification of all infectious agents in the database used to interpret the obtained MS data. The ability to rapidly identify even rare infectious agents, allows for a faster awareness. This can aid in adequate treatment planning tailored towards the identified infectious agent. Furthermore, this can facilitate prevention of spreading of infectious diseases.

A possible drawback of the presented study is that during the validation of the LC-MS/MS method sheep erythrocytes were used as a surrogate for whole blood. However, a recent publication presented a similar LC-MS/MS based method, including a comparable saponin based sample preparation, that was functional on clinical positive blood cultures [32]. Moreover, despite matrix derived peptides from whole blood and culture media were identified, there were enough discriminative peptides identified that enabled the identification of the expressed extended spectrum $\beta$-lactamases [32].

The LC-MS/MS method presented in this study is generic for the identification of microorganisms from positive blood cultures, but can also be used for all type of microorganisms on or in cultured (liquid) media. Recent publications demonstrated that nontargeted LC-MS/MS-based approaches could analyze the susceptibility against $\beta$-lactam-based 
antibiotics [32,33] in Gram-negatives. Next, by using a targeted approach (SRM mass spectrometry) was possible to detect methicillin resistance in $S$. aureus and virulence factors directly from positive blood cultures [34]. Furthermore, by the detection of amino acid substitutions in the GyrA protein using SRM it was possible to detect decreased quinolone susceptibility in typhoidal Salmonella isolates [35]. The combination of identification with antibiotic resistance determination will directly benefit clinical management.

\section{Conclusion \& future perspective}

A generic LC-MS/MS method, based on peptide sequences, was developed that rapidly identifies microorganisms in positive blood cultures. Tandem mass spectrometry technology has improved to a point in which it can improve clinical microbiology diagnostics. With the introduction of high-resolution hybrid mass spectrometers, both targeted and nontargeted analysis of the proteome can be combined, allowing unbiased screening with limited or no loss of sensitivity [36]. It is common practice in clinical microbiology to determine the analytical strategy to identify pathogens and antimicrobial susceptibility based on the interpretation of the Gramstaining. In the future there is no need to execute this type of differential diagnostics on positive blood cultures. By measuring the proteome using high-resolution hybrid mass spectrometers and the presented approach, microorganisms grown in blood culture flasks can be identified unambiguously. Additionally, based on the identification of the microbial agent, the recorded MS data can be searched for specific peptide sequences of interest such as peptides that reveal decreased susceptibility, increased virulence or peptides that are epidemiological markers of an outbreak strain. Before LC-MS/MS-based diagnostics can be implemented as a routine analysis in clinical microbiology, a fully automated sample preparation and dedicated microbiology data analysis software have to be developed. These developments in combination with further customization of mass spectrometry systems, will lead to the possibility of a fully automated pipeline of blood culture analysis in the future.

\section{Financial \& competing interests disclosure}

This work was funded by the Dutch Ministry of Defence under grant number V1408. The authors have no other relevant affiliations or financial involvement with any organization or entity with a financial interest in or financial conflict with the subject matter or materials discussed in the manuscript apart from those disclosed.

No writing assistance was utilized in the production of this manuscript.

\section{Ethical conduct of research}

The authors state that they have obtained appropriate institutional review board approval or have followed the principles outlined in the Declaration of Helsinki for all human or animal experimental investigations. In addition, for investigations involving human subjects, informed consent has been obtained from the participants involved.

\section{Open access}

This work is licensed under the AttributionNonCommercial-NoDerivatives 4.0 Unported License. To view a copy of this license, visit http://creativecommons.org/ licenses/by-nc-nd/4.0/

\section{Supplementary data}

To view the supplementary data that accompany this paper please visit the journal website at: www.futuremedicine. com/doi/full/10.2217/fmb-2017-0050.

\section{SUMMARY POINTS}

\section{Background}

- Accurate identification of the causative pathogen(s) of bloodstream infections guides optimal treatment.

\section{Results}

- A sample preparation was developed for the direct identification of bacteria grown in blood culture flasks using liquid chromatography-tandem mass spectrometry (LC-MS/MS).

- The developed data analysis for LC-MS/MS data enabled accurate identification to species level of grown microorganisms, based on discriminative peptides.

- Microorganisms were correctly identified by LC-MS/MS in $100 \%$ of the monomicrobial blood cultures and in $78 \%$ of the polymicrobial blood cultures.

\section{Conclusion}

- The presented LC-MS/MS method enables generic and accurate identification of pathogen(s) grown in blood culture flasks. 


\section{References}

1 Seifert $\mathrm{H}$. The clinical importance of microbiological findings in the diagnosis and management of bloodstream infections. Clin. Infect. Dis. 48(Suppl. 4), S238-S245 (2009).

2 Koupetori M, Retsas T, Antonakos N et al. Bloodstream infections and sepsis in Greece: over-time change of epidemiology and impact of de-escalation on final outcome. $B M C$ Infect. Dis. 14(1), 1-10 (2014).

3 Anderson DJ, Moehring RW, Sloane R et al. Bloodstream infections in community hospitals in the 21st century: a multicenter cohort study. PLoS ONE 9(3), e91713 (2014).

4 Ammerlaan HSM, Harbarth S, Buiting AGM et al. Secular trends in nosocomial bloodstream infections: antibiotic-resistant bacteria increase the total burden of infection. Clin. Infect. Dis. 56(6), 798-805 (2013).

5 Wang MC, Lin WH, Yan JJ et al. Early identification of microorganisms in blood culture prior to the detection of a positive signal in the BACTEC FX system using matrix-assisted laser desorption/ionizationtime of flight mass spectrometry. J. Microbiol. Immunol. Infec. 48(4), 419-424 (2015).

6 Peters RPH, Van Agtmael MA, Danner SA, Savelkoul PHM, VandenbrouckeGrauls CMJE. New developments in the diagnosis of bloodstream infections. Lancet Infect. Dis. 4(12), 751-760 (2004).

7 Bouza E, Burillo A, Muñoz P, Guinea J, Marín M, Rodríguez-Créixems M. Mixed bloodstream infections involving bacteria and Candida spp. J. Antimicrob. Chemother. 68(8), 1881-1888 (2013).

8 Sancho S, Artero A, Zaragoza R, Camarena JJ, Gonzã ¡Lez R, Nogueira JM. Impact of nosocomial polymicrobial bloodstream infections on the outcome in critically ill patients. Eur. J. Clin. Microbiol. Infect. Dis. 31(8), 1791-1796 (2012).

9 Vlek ALM, Bonten MJM, Boel CHE. Direct matrix-assisted laser desorption ionization time-of-flight mass spectrometry improves appropriateness of antibiotic treatment of bacteremia. PLoS ONE 7(3), e32589 (2012).

10 Deresinski S. Principles of antibiotic therapy in severe infections: optimizing the therapeutic approach by use of laboratory and clinical data. Clin. Infect. Dis. 45(Suppl. 3), S177-S183 (2007).

11 Tan KE, Ellis BC, Lee R, Stamper PD, Zhang SX, Carroll KC. Prospective evaluation of a matrix-assisted laser desorption ionization-time of flight mass spectrometry system in a hospital clinical microbiology laboratory for identification of bacteria and yeasts: a bench-by-bench study for assessing the impact on time to identification and cost-effectiveness. J. Clin. Microbiol. 50(10), 3301-3308 (2012).

12 Steensels D, Verhaegen J, Lagrou K. Matrix-assisted laser desorption ionizationtime of flight mass spectrometry for the identification of bacteria and yeasts in a clinical microbiological laboratory: a review. Acta Clin. Belg. 66(4), 267-273 (2011).

13 Tadros M, Petrich A. Evaluation of MALDI-TOF mass spectrometry and Sepsityper $\mathrm{Kit}^{\mathrm{TM}}$ for the direct identification of organisms from sterile body fluids in a Canadian pediatric hospital. Can. J. Infect. Dis. Med. Microbiol. 24(4), 191-194 (2013).

14 Mahé P, Arsac M, Chatellier S et al. Automatic identification of mixed bacterial species fingerprints in a MALDI-TOF mass-spectrum. Bioinformatics 30 (9), 1280-1286 (2014).

15 Tracz DM, Mccorrister SJ, Chong PM, Lee DM, Corbett CR, Westmacott GR. A simple shotgun proteomics method for rapid bacterial identification. J. Microbiol. Methods 94(1), 54-57 (2013).

16 Dworzanski JP, Deshpande SV, Chen R et al. Mass spectrometry-based proteomics combined with bioinformatic tools for bacterial classification. J. Proteome Res. 5(1), 76-87 (2006).

17 Jabbour RE, Deshpande SV, Wade MM et al. Double-blind characterization of non-genomesequenced bacteria by mass spectrometrybased proteomics. Appl. Environ. Microbiol. 76(11), 3637-3644 (2010).

18 Majchrzykiewicz-Koehorst JA, Heikens E, Trip $\mathrm{H}$ et al. Rapid and generic identification of influenza $\mathrm{A}$ and other respiratory viruses with mass spectrometry. J. Virol. Methods 213, 75-83 (2015).

19 Jorakate P, Higdon M, Kaewpan A et al. Contribution of the BacT/Alert MB Mycobacterium bottle to bloodstream infection surveillance in Thailand: added yield for Burkholderia pseudomallei. J. Clin. Microbiol. 53(3), 910-914 (2015).

20 Jo SJ, Park KG, Han K, Park DJ, Park YJ. Direct identification and antimicrobial susceptibility testing of bacteria from positive blood culture bottles by matrix-assisted laser desorption/ionization time-of-flight mass spectrometry and the VITEK 2 system. Ann. Lab. Med. 36(2), 117-123 (2016).

21 Ma B, Zhang K, Hendrie C et al. PEAKS: powerful software for peptide de novo sequencing by tandem mass spectrometry. Rapid. Commun. Mass Spectrom. 17(20), 2337-2342 (2003).
22 Loonen AJM, Wolffs PFG, Bruggeman CA, Van DB. Developments for improved diagnosis of bacterial bloodstream infections. Eur. J. Clin. Microbiol. Infect. Dis. 33(10), 1687-1702 (2014).

23 Lagacé-Wiens PRS, Adam HJ, Karlowsky JA et al. Identification of blood culture isolates directly from positive blood cultures by use of matrix-assisted laser desorption ionizationtime of flight mass spectrometry and a commercial extraction system: analysis of performance, cost, and turnaround time. J. Clin. Microbiol. 50(10), 3324-3328 (2012).

24 Kok J, Thomas LC, Olma T, Chen SCA, Iredell JR. Identification of bacteria in blood culture broths using matrix-assisted laser desorption-ionization Sepsityper ${ }^{\mathrm{TM}}$ and time of flight mass spectrometry. PLoS ONE 6(8), e23285 (2011).

25 La Scola B, Raoult D. Direct identification of bacteria in positive blood culture bottles by matrix-assisted laser desorption ionisation time-of-flight mass spectrometry. PLoS ONE 4(11), e8041 (2009).

26 Yonetani S, Ohnishi H, Ohkusu K, Matsumoto T, Watanabe T. Direct identification of microorganisms from positive blood cultures by MALDI-TOF MS using an in-house saponin method. Int. J. Infect. Dis. 52, 37-42 (2016).

27 Pupo GM, Lan R, Reeves PR. Multiple independent origins of Shigella clones of Escherichia coli and convergent evolution of many of their characteristics. Proc. Natl Acad. Sci. USA 97(19), 10567-10572 (2000).

28 Ogura Y, Ooka T, Iguchi A et al. Comparative genomics reveal the mechanism of the parallel evolution of $\mathrm{O} 157$ and non-O157 enterohemorrhagic Escherichia coli. Proc. Natl Acad. Sci. USA 106(42), 17939-17944 (2009).

29 Lan R, Alles MC, Donohoe K, Martinez MB, Reeves PR. Molecular evolutionary relationships of enteroinvasive Escherichia coli and Shigella spp. Infect. Immun. 72(9), 5080-5088 (2004).

30 Touchon M, Hoede C, Tenaillon O et al. Organised genome dynamics in the Escherichia coli species results in highly diverse adaptive paths. PLoS Genet. 5(1), e1000344 (2009).

31 Bergey's Manual of Systematic Bacteriology, Volume 3: The Firmicutes (2nd Edition). Vos P, Garrity G, Jones D et al.. (Eds). Springer-Verlag New York, Berlin, Germany (2009).

32 Fleurbaaij F, Goessens W, Van Leeuwen HC et al. Direct detection of extended-spectrum beta-lactamases (CTX-M) from blood 
cultures by LC-MS/MS bottom-up proteomics. Eur. J. Clin. Microbiol. Infect. Dis. doi:10.1007/s10096-017-2975-y (2017) (Epub ahead of print).

33 Trip H, Mende K, MajchrzykiewiczKoehorst JA et al. Simultaneous identification of multiple beta-lactamases in Acinetobacter baumannii in relation to carbapenem and ceftazidime resistance, using liquid chromatography-tandem mass spectrometry. J. Clin. Microbiol. 53(6), 1927-1930 (2015).

34 Charretier Y, Dauwalder O, Franceschi C et al. Rapid bacterial identification, resistance, virulence and type profiling using selected reaction monitoring mass spectrometry. Sci. Rep. 5, 13944 (2015).

35 Hassing RJ, Goessens WH, Zeneyedpour L et al. Detection of amino acid substitutions in the GyrA protein of fluoroquinolone-resistant typhoidal Salmonella isolates using highresolution mass spectrometry. Int. J. Antimicrob. Agents 47(5), 351-356 (2016).

36 Peterson AC, Russell JD, Bailey DJ, Westphall MS, Coon JJ. Parallel reaction monitoring for high resolution and high mass accuracy quantitative, targeted proteomics. Mol. Cell. Proteomics 11(11), 1475-1488 (2012). 